\title{
Modeling of Magnetoelectric Interaction in Magnetostrictive-Piezoelectric Composites
}

\author{
M. I. Bichurin and V. M. Petrov \\ Institute of Electronic and Information Systems, Novgorod State University, 173003 Veliky Novgorod, Russia
}

Correspondence should be addressed to V. M. Petrov, vladimir.petrov@novsu.ru

Received 12 September 2011; Accepted 22 November 2011

Academic Editor: Shashank Priya

Copyright (C 2012 M. I. Bichurin and V. M. Petrov. This is an open access article distributed under the Creative Commons Attribution License, which permits unrestricted use, distribution, and reproduction in any medium, provided the original work is properly cited.

\begin{abstract}
The paper dwells on the theoretical modeling of magnetoelectric (ME) effect in layered and bulk composites based on magnetostrictive and piezoelectric materials. Our analysis rests on the simultaneous solution of elastodynamic or elastostatic and electro/magnetostatic equations. The expressions for ME coefficients as the functions of material parameters and volume fractions of components are obtained. Longitudinal, transverse, and in-plane cases are considered. The use of the offered model has allowed to present the ME effect in ferrite cobalt-barium titanate, ferrite cobalt-PZT, ferrite nickel-PZT, and lanthanum strontium manganite-PZT composites adequately.
\end{abstract}

\section{Introduction}

The magnetoelectric (ME) effect in a material consists in inducing the electric polarization in an applied magnetic field, or vice versa, inducing the magnetization in an applied electric field. The effect was first observed in antiferromagnetic $\mathrm{Cr}_{2} \mathrm{O}_{3}$ with a room temperature ME voltage coefficient of $20 \mathrm{mV} / \mathrm{cm}$ Oe [1]. Using magnetostrictive-piezoelectric composites gives rise to an enhancement of ME effects [2,3]. A composite is expected to be magnetoelectric since the ME coupling is the product of the magnetostrictive deformation and the piezoelectric field generation [4-6]. The ME voltage coefficient $\alpha_{E}=\delta E / \delta H$ where $\delta H$ and $\delta E$ are the applied magnetic and induced electric AC fields, correspondingly. Lead zirconate titanate- (PZT-) ferrite and PZT-Terfenol$\mathrm{D}$ are the most studied composites to date $[7,8]$. One of largest ME voltage coefficients of $500 \mathrm{~V} /(\mathrm{cm} \cdot \mathrm{Oe})$ was reported recently for a high permeability magnetostrictive piezofiber laminate [9].

The thorough investigation of ME materials is stipulated by potential application for novel devices. In our earlier works, ME effect has been studied for low-frequency, electromechanical resonance (EMR) and magnetic resonance (FMR) regions. In addition, we have first studied ME interaction at the coincidence of EMR and FMR, that is, magneto-acoustic resonance (MAR). In this paper, we discuss detailed mathematical modeling approaches that are used to describe the dynamic behavior of ME coupling in magnetostrictive-piezoelectric composites at low-frequencies and in EMR region. Expressions for ME coefficients are obtained using the solution of elastostatic/elastodynamic and electrostatic/magnetostatic equations. The ME voltage coefficients are estimated from the known material parameters. The basic methods developed for decreasing the resonance frequencies are analyzed.

\section{Modeling of Magnetoelectric Effect in Low-Frequency Region}

2.1. Symmetric Layered Structures. Using layered structures enables one to overcome a series of difficulties that are characteristic for bulk composites. The reasons for the giant ME effects in layered composites are (a) high piezoelectric and piezomagnetic coefficients in individual layers, (b) effective stress transfer between layers, (c) ease of poling and subsequent achievement of a full piezoelectric effect, and (d) ability to hold charge due to suppression of leakage currents across composites with a 2-2 connectivity. 
Prior theoretical models based on mechanics and constitutive relationships by Harshe et al. [10] were restricted to account for longitudinal ME voltage coefficient in laminates having ideal mechanical connection at the interfaces between layers. Principal disadvantages of this earlier approach [10] were as follows: (i) for the case of longitudinally oriented fields, the effect of the magnetic permeability of the ferrite phase was ignored. Diminution of interior (local) magnetic fields results in a weakening of ME interactions via demagnetization fields. (ii) The case of fields applied in crossorientations to the ME layer connectivity was not considered, in which later experimental investigations revealed large $\mathrm{ME}$ responses.

In this work, we present a summary of a more recent theory of ME laminate composites, which are free from the disadvantages mentioned just above. The approach is based on continuum mechanics, and considers the composite as a homogeneous medium having piezoelectric and magnetostrictive subsystems. To derive the effective material parameters of composites, an averaging method consisting of two steps [11-13] should be used. In the first step, the composite is considered as a structure whose magnetostrictive and piezoelectric phases are distinct and separable. ME composites are characterized by the presence of magnetic and electric subsystems interacting with each other.

The constitutive equation for the piezoelectric effect can be given in the following form:

$$
\begin{gathered}
{ }^{p} S_{i}={ }^{p} s_{i j}{ }^{p} T_{j}+{ }^{p} d_{k i}{ }^{p} E_{k}, \\
{ }^{p} D_{k}={ }^{p} d_{k i}{ }^{p} T_{i}+{ }^{p} \varepsilon_{k n}{ }^{p} E_{n},
\end{gathered}
$$

where ${ }^{p} S_{i}$ is a strain tensor component of the piezoelectric phase; ${ }^{p} E_{k}$ is a vector component of the electric field; ${ }^{p} D_{k}$ is a vector component of the electric displacement; ${ }^{p} T_{i}$ is a stress tensor component of the piezoelectric phase; ${ }^{p} s_{i j}$ is a compliance coefficient of the piezoelectric phase; ${ }^{p} d_{k i}$ is a piezoelectric coefficient of the piezoelectric phase; ${ }^{p} \varepsilon_{k n}$ is a permittivity matrix of the piezoelectric phase.

Analogously, the strain and magnetic induction tensors of the magnetostrictive phase are, respectively,

$$
\begin{aligned}
& { }^{m} S_{i}={ }^{m} s_{i j}{ }^{m} T_{j}+{ }^{m} q_{k i}{ }^{m} H_{k}, \\
& { }^{m} B_{k}={ }^{m} q_{k i}{ }^{m} T_{i}+{ }^{m} \mu_{k n}{ }^{m} H_{n},
\end{aligned}
$$

where ${ }^{m} S_{i}$ is a strain tensor component of the magnetostrictive phase; ${ }^{m} T_{j}$ is a stress tensor component of the magnetostrictive phase; ${ }^{m} s_{i j}$ is a compliance coefficient of the magnetostrictive phase; ${ }^{m} H_{k}$ is a vector component of magnetic field; ${ }^{m} B_{k}$ is a vector component of magnetic induction; ${ }^{m} q_{k i}$ is a piezomagnetic coefficient; ${ }^{m} \mu_{k n}$ is a permeability matrix.

Prior models assumed that the connection at interfaces between layers was ideal. However, in this chapter, we assume that there is a coupling parameter $k=$ $\left({ }^{p} S_{i}-{ }^{p} S_{i 0}\right) /\left({ }^{m} S_{i}-{ }^{p} S_{i 0}\right)(i=1,2)$, where ${ }^{p} S_{i 0}$ is a strain tensor component assuming no friction between layers [13]. This interphase-interface elastic-elastic coupling parameter

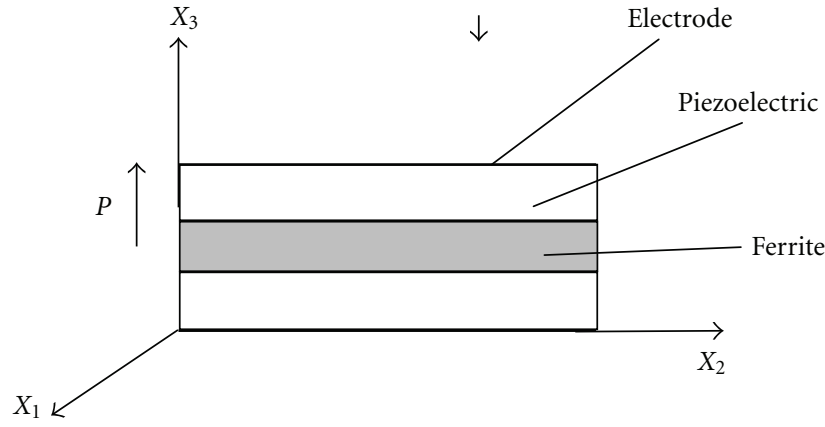

FIGURE 1: Schematic of the layered composite structure.

depends on interface quality, and it is a measure of a differential deformation between piezoelectric and magnetostrictive layers. The coupling parameter is $k=1$ for the case of an ideal interface, and is $k=0$ for the case of no friction.

In the second step of the averaging method to derive the effective materials parameters, the bilayer composite is considered as a homogeneous solid, whose behavior can be described by the following coupled sets of linear algebraic equations:

$$
\begin{gathered}
S_{i}=s_{i j} T_{j}+d_{k i} E_{k}+q_{k i} H_{k}, \\
D_{k}=d_{k i} T_{i}+\varepsilon_{k n} E_{n}+\alpha_{k n} H_{n}, \\
B_{k}=q_{k i} T_{i}+\alpha_{k n} E_{n}+\mu_{k n} H_{n},
\end{gathered}
$$

where $S_{i}$ is a strain tensor component; $T_{j}$ is a stress tensor component; $E_{k}$ is a vector component of the electric field; $D_{k}$ is a vector component of the electric displacement; $H_{k}$ is a vector component of the magnetic field; $B_{k}$ is a vector component of the magnetic induction; $s_{i j}$ is an effective compliance coefficient; $d_{k i}$ is a piezoelectric coefficient; $q_{k i}$ is a piezomagnetic coefficient; $\varepsilon_{k n}$ is an effective permittivity; $\mu_{k n}$ is a permeability coefficient; $\alpha_{k n}$ is a ME coefficient.

The simultaneous solution of the coupled sets of linear algebraic equations allows one to find the effective parameters of a composite.

Let us consider that the layers of a composite are oriented along the planes $\left(X_{1}, X_{2}\right)$, and that the direction $X_{3}$ is perpendicular to the same plane. In this case, the direction of polarization in a sample coincides with the $X_{3}$ axis. If we by choice apply a constant magnetic bias and variable magnetic field along the same direction coincidental with that of the polarization, then any resultant electric field will also be parallel to the $X_{3}$ axis, as shown in Figure 1. This summarization supposes that the symmetry of the piezoelectric phase is $\infty \mathrm{m}$, and that of the magnetic phase is cubic. The following boundary conditions can then be used to derive expressions for ME coefficients:

$$
\begin{gathered}
{ }^{p} S_{i}=k^{m} S_{i}+(1-k){ }^{p} S_{i 0} ; \quad(i=1,2), \\
{ }^{p} T_{i}=-\frac{{ }^{m} T_{i}(1-v)}{v} ; \quad(i=1,2),
\end{gathered}
$$


where $v=p v /\left(p v+{ }^{m} v\right)$ and $p v$ and ${ }^{m} v$ denote the Poisson's ratio of the piezoelectric and magnetostrictive phases, respectively; ${ }^{p} S_{10}$ and ${ }^{p} S_{20}$ are the strain tensor components for $k=0$.
Using continuity conditions for magnetic and electric fields, and using open circuit condition, one can then obtain the following expressions for the ME susceptibility, and longitudinal ME voltage coefficient.

$$
\begin{aligned}
\alpha_{33}=2 & \frac{k \mu_{0}(v-1)^{p} d_{31}{ }^{m} q_{31}}{\left[\mu_{0}(v-1)-{ }^{m} \mu_{33} v\right]\left[k v\left({ }^{m} s_{12}+{ }^{m} s_{11}\right)-\left({ }^{p} s_{11}+{ }^{p} s_{12}\right)(v-1)\right]+2^{m} q_{31}^{2} k v^{2}}, \\
\alpha_{E, 33}=\frac{E_{3}}{H_{3}}= & 2 \frac{\mu_{0} k v(1-v){ }^{p} d_{31}{ }^{m} q_{31}}{\left\{2^{p} d_{31}^{2}(1-v)+{ }^{p} \varepsilon_{33}\left[\left({ }^{p} s_{11}+{ }^{p} s_{12}\right)(v-1)-v\left({ }^{m} s_{11}+{ }^{m} s_{12}\right)\right]\right\}} \\
& \times \frac{\left[\left({ }^{p} s_{11}+{ }^{p} s_{12}\right)(v-1)-k v\left({ }^{m} s_{11}+{ }^{m} s_{12}\right)\right]}{\left\{\left[\mu_{0}(v-1)-{ }^{m} \mu_{33} v\right]\left[k v\left({ }^{m} s_{12}+{ }^{m} s_{11}\right)-\left({ }^{p} s_{11}+{ }^{p} s_{12}\right)(v-1)\right]+2^{m} q_{31}^{2} k v^{2}\right\}} .
\end{aligned}
$$

The earlier expression obtained by Harshe et al. [10] matched our theory for the special case of $k=1$, provided that the magnetic field is applied only to the ferrite phase.

The model presented above allows for the determination of the longitudinal ME coefficients as functions of volume fractions, physical parameters of phases, and elastic-elastic interfacial coupling parameter $k$.

Next we consider the transverse fields' orientation that corresponds to $E$ and $\delta E$ being applied along the $X_{3}$ direction, and $H$ and $\delta H$ along the $X_{1}$ direction (in the sample plane). The expressions for the ME susceptibility, and transverse ME voltage coefficient are then, respectively,

$$
\begin{aligned}
& \alpha_{31}=\frac{(v-1) v\left({ }^{m} q_{11}+{ }^{m} q_{21}\right){ }^{p} d_{31} k}{(v-1)\left({ }^{p} s_{11}+{ }^{p} s_{12}\right)-k v\left({ }^{m} s_{11}+{ }^{m} s_{12}\right)}, \\
& \alpha_{E, 31} \\
& =\frac{E_{3}}{H_{1}} \\
& =\frac{-k v(1-v)\left({ }^{m} q_{11}+{ }^{m} q_{21}\right){ }^{p} d_{31}}{p_{\mathcal{E}_{33}}\left({ }^{m} s_{12}+{ }^{m} s_{11}\right) k v+{ }^{p} \mathcal{E}_{33}\left({ }^{p} s_{11}+{ }^{p} s_{12}\right)(1-v)-2 k^{p} d_{31}^{2}(1-v)} .
\end{aligned}
$$

Finally, we consider a bilayer laminate that is poled with an electric field $E$ in the plane of the sample. We suppose that the in-plane fields $H$ and $\delta H$ are parallel, and that the induced electric field $\delta E$ is measured in the same direction (i.e., along the $c$-axis). Expressions for the $\alpha$, and $\alpha_{E}^{\prime}$ can be obtained in the following form:

$$
\begin{aligned}
\alpha_{11}= & \left\{\left[{ }^{m} q_{11}\left({ }^{p} s_{33}{ }^{p} d_{11}-{ }^{p} s_{12}{ }^{p} d_{12}\right)\right.\right. \\
& \left.+{ }^{m} q_{12}\left({ }^{p} s_{11}{ }^{p} d_{12}-{ }^{p} s_{12}{ }^{p} d_{11}\right)\right](1-v) \\
& +\left[{ }^{m} q_{11}\left({ }^{m} s_{11}{ }^{p} d_{11}-{ }^{m} s_{12}{ }^{p} d_{12}\right)\right. \\
& \left.\left.\quad+{ }^{m} q_{12}\left({ }^{m} s_{11}{ }^{p} d_{12}-{ }^{m} s_{12}{ }^{p} d_{11}\right)\right] v k\right\} v k(1-v) / \\
& {\left[\left({ }^{p} s_{33}{ }^{p} s_{11}-{ }^{p} s_{12}{ }^{2}\right)(1-v)^{2}+k^{2} v^{2}\left({ }^{m} s_{11}{ }^{2}-{ }^{m} s_{12}{ }^{2}\right)\right.} \\
& \left.+\left({ }^{p} s_{33}{ }^{m} s_{11}+{ }^{m} s_{11}{ }^{p} s_{11}-2^{m} s_{12}{ }^{p} s_{13}\right) k v(1-v)\right],
\end{aligned}
$$

$$
\begin{aligned}
\alpha_{E, 11}= & \left(\left({ }^{m} q_{11}\left({ }^{p} s_{33}{ }^{p} d_{11}-{ }^{p} s_{12}{ }^{p} d_{12}\right)\right.\right. \\
& \left.+{ }^{m} q_{12}\left({ }^{p} s_{11}{ }^{p} d_{12}-{ }^{p} s_{12}{ }^{p} d_{11}\right)\right)(1-v) \\
& +\left({ }^{m} q_{11}\left({ }^{m} s_{11}{ }^{p} d_{11}-{ }^{m} s_{12}{ }^{p} d_{12}\right)\right. \\
& \left.\left.+{ }^{m} q_{12}\left({ }^{m} s_{11}{ }^{p} d_{12}-{ }^{m} s_{12}{ }^{p} d_{11}\right)\right) v k\right) v k(1-v) / \\
& \left(\left((1-p)^{m} \varepsilon_{11}+v^{p} \varepsilon_{11}\right)\right. \\
& \times\left((1-v)^{2}\left({ }^{p} s_{11}{ }^{p} s_{33}-{ }^{p} s_{12}{ }^{2}\right)+(1-v) v k\right. \\
& \quad \times\left({ }^{m} s_{11}{ }^{p} s_{11}+{ }^{p} s_{33}{ }^{m} s_{11}-2{ }^{p} s_{12}{ }^{m} s_{12}\right) \\
& \left.+k^{2} v^{2}\left({ }^{m} s_{11}{ }^{2}-{ }^{m} s_{12}{ }^{2}\right)\right) \\
- & k v(1-p)^{2}\left(2^{p} s_{12}{ }^{p} d_{11}{ }^{p} d_{12}-{ }^{p} s_{33}{ }^{p} d_{11}{ }^{2}\right. \\
- & \left.{ }^{p} s_{11}{ }^{p} d_{12}{ }^{2}\right)+k^{2} v^{2}(1-v) \\
& \left.\times\left({ }^{m} s_{11}{ }^{p} d_{12}{ }^{2}+{ }^{m} s_{11}{ }^{p} d_{11}{ }^{2}-2^{m} s_{12}{ }^{p} d_{11} p d_{11}\right)\right) .
\end{aligned}
$$

Amongst all the cases presented so far, the in-plane ME coefficient is expected to be the largest. This is due to availability of magnetostrictive and piezoelectric phases with high $q$ - and $d$-values, respectively, and, to the absence of demagnetization fields. We will further use these outcomes later in the estimation of ME parameters for some specific examples.

2.2. Bilayer Structure. Theoretical modeling of lowfrequency $\mathrm{ME}$ effect described above is based on the homogeneous longitudinal strain approach. However, configurational asymmetry of a bilayer implies bending the sample in applied magnetic or electric field and variation in $\mathrm{ME}$ response. One of the principal objective of present section is modeling of the $\mathrm{ME}$ interaction in a magnetostrictive-piezoelectric bilayer taking into account the flexural strains [14]. We calculated ME voltage coefficients $\alpha_{E}$ for transverse field orientations to provide minimum demagnetizing fields and maximum $\alpha_{E}$. 
TABle 1: Material parameters (compliance coefficient $s$, piezomagnetic coupling $q$, piezoelectric coefficient $d$, and permittivity $\varepsilon$ ) for lead zirconate titanate (PZT), cobalt ferrite (CFO), and lanthanum strontium manganite used for theoretical values.

\begin{tabular}{lccccccccc}
\hline Material & $\begin{array}{c}s_{11} \\
\left(10^{-12} \mathrm{~m}^{2} / \mathrm{N}\right)\end{array}$ & $\begin{array}{c}s_{12} \\
\left(10^{-12} \mathrm{~m}^{2} / \mathrm{N}\right)\end{array}$ & $\begin{array}{c}s_{13} \\
\left(10^{-12} \mathrm{~m}^{2} / \mathrm{N}\right)\end{array}$ & $\begin{array}{c}s_{33} \\
\left(10^{-12} \mathrm{~m}^{2} / \mathrm{N}\right)\end{array}$ & $\begin{array}{c}q_{33} \\
\left(10^{-12} \mathrm{~m} / \mathrm{A}\right)\end{array}$ & $\begin{array}{c}q_{31} \\
\left(10^{-12} \mathrm{~m} / \mathrm{A}\right)\end{array}$ & $\begin{array}{c}d_{31} \\
\left(10^{-12} \mathrm{~m} / \mathrm{V}\right)\end{array}$ & $\begin{array}{c}d_{33} \\
\left(10^{-12} \mathrm{~m} / \mathrm{V}\right)\end{array}$ & $\varepsilon_{33} / \varepsilon_{0}$ \\
\hline PZT & 15.3 & -5 & -7.22 & 17.3 & - & - & -175 & 400 \\
CFO & 6.5 & -2.4 & & & -1880 & 556 & - & - \\
LSMO & 15 & -5 & & & 250 & -120 & - & - \\
\hline
\end{tabular}

The thickness of the plate is assumed small compared to remaining dimensions. We assume the longitudinal axial strains of each layer to be linear functions of the vertical coordinate $z_{i}$ to take into account bending the sample. To preserve force equilibrium, the axial forces in the three layers add up to zero, that is,

$$
F_{p 1}+F_{m 1}=0
$$

where $F_{i 1}=\int_{-}^{i}{ }^{i} t / 2{ }^{i} T_{1} d z_{1}$.

The moment equilibrium condition has the form:

$$
F_{m 1} h_{m}=M_{m 1}+M_{p 1}
$$

where $M_{i 1}=\int_{-}^{i}{ }^{i} t / 2{ }_{t} z_{i}{ }^{i} T_{1} d z_{1}$.

Simultaneous solving (8) and (9) enables finding the axial stress components in the piezoelectric layer ${ }^{p} T_{1}$ and ${ }^{p} T_{2}$. Then the expression for ME voltage coefficient can be expressed using the open circuit condition

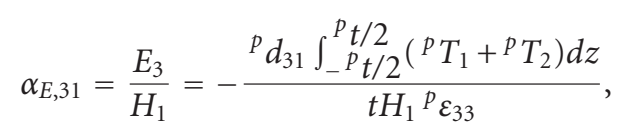

where $t={ }^{m} t+{ }^{p} t+{ }^{s} t$ is the total thickness of considered structure.

In case of neglecting the flexural strains, it is easily shown that expression for ME voltage coefficient reduces to wellknown expression of Section 2.1 which was obtained with the assumption of homogeneous longitudinal strains.

2.3. Examples of Multilayer Structures. The preceding comprehensive theoretical treatment resulted in expressions of the ME voltage coefficients for three different orientations of fields, which were the ones of most importance, including longitudinal, transverse, and in-plane longitudinal. The most significant features of the model are as follows: (i) Consideration of three different field configurations. This allows for the determination of a single-valued interface parameter $k$, facilitating quantitative characterization of the bilayer interface. (ii) Consideration of a new field configuration, that is, in-plane longitudinal fields that has very strong $\mathrm{ME}$ coupling. (iii) consideration of the effect of a finite magnetic permeability on the magnetostriction of the magnetic subsystem: which was ignored in prior investigations.

Next, we apply the theory for the calculation of ME coupling in layered composites. Consider the materials couple cobalt ferrite and lead zirconate titanate (CFO-PZT),

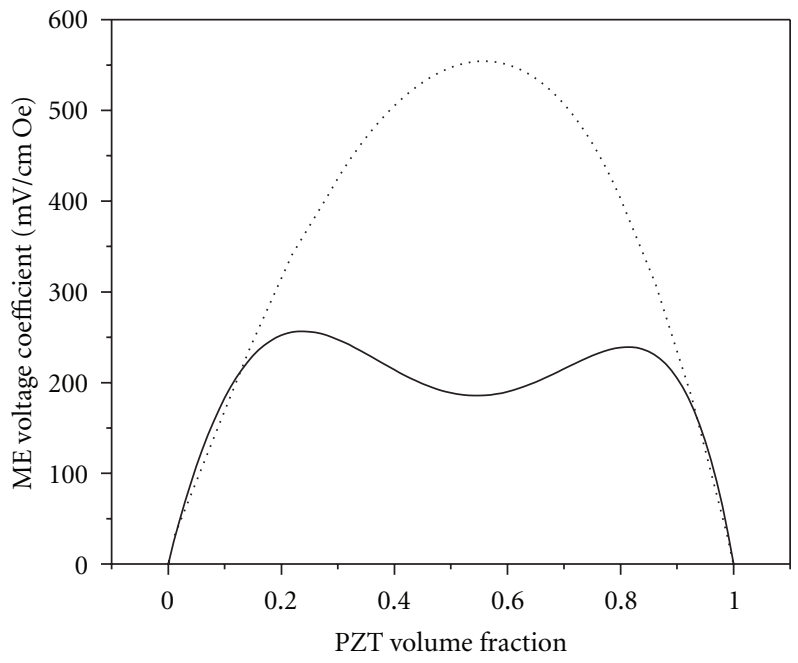

Figure 2: Transverse ME voltage coefficient $\alpha_{E, 31}=\delta E_{3} / \delta H_{1}$ for a perfectly bonded $(k=1)$ bilayer (solid line) and symmetric structure (dot line) of CFO and PZT.

which is a system that has been of significant prior interests. Since the value of $\alpha_{E}$ depends notably on the concentration of the two phases, the ME voltage coefficient has been determined as a function of the volume fraction $v$ of the piezoelectric phase in composite. Material parameters used for estimates are given in Table 1. Results of calculations using the model are illustrated in Figure 2, which were obtained by assuming an ideal interface coupling $(k=1)$.

Results of $\alpha_{E, 31}$ versus PZT volume fraction reveals a double maximum that is due to fact that the strain produced by the ferrite consists of two components: longitudinal and flexural. For a symmetric structure such as trilayer, there are no flexural strains and the maximum ME coefficient occurs for $V=0.6$ [13]. Since the flexural strain is opposite in sign compared to longitudinal one and reaches its maximum value for $V=0.6$, the two types of strains combine to produce suppression of $\alpha_{E, 31}$ at $V=0.6$ and a double maximum in the $\mathrm{ME}$ coefficient as in Figure 2. In what follows, we consider theoretical models of low frequency ME coupling for symmetric structures taking into account that such structures are described by higher values of $\mathrm{ME}$ coefficients.

The variation of $\alpha_{E, 33}$ with $v$ for various values of coupling parameter $k$ is shown in Figure 3(a) for a symmetric structure which excludes the flexural deformations. The magnitude of $\alpha_{E, 33}$ decreases with decreasing $k$, and 


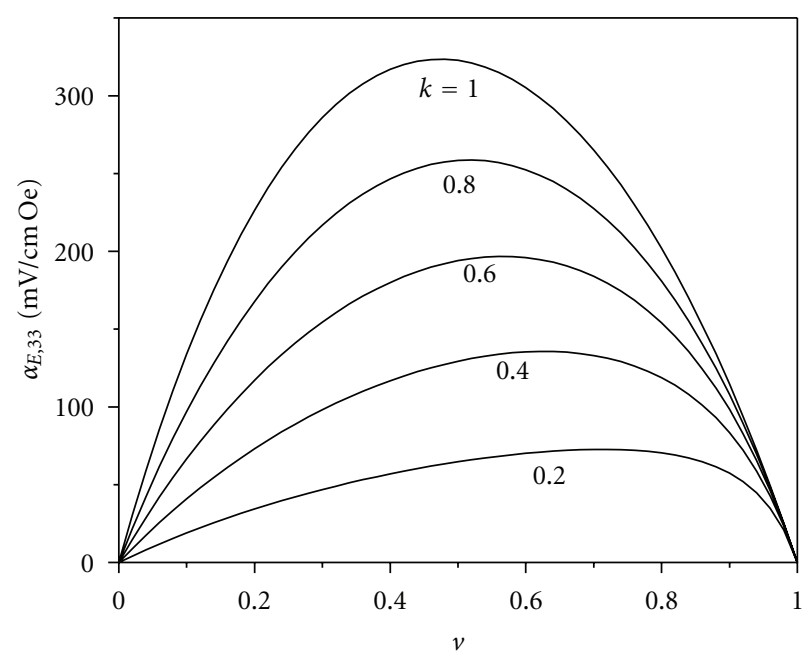

(a)

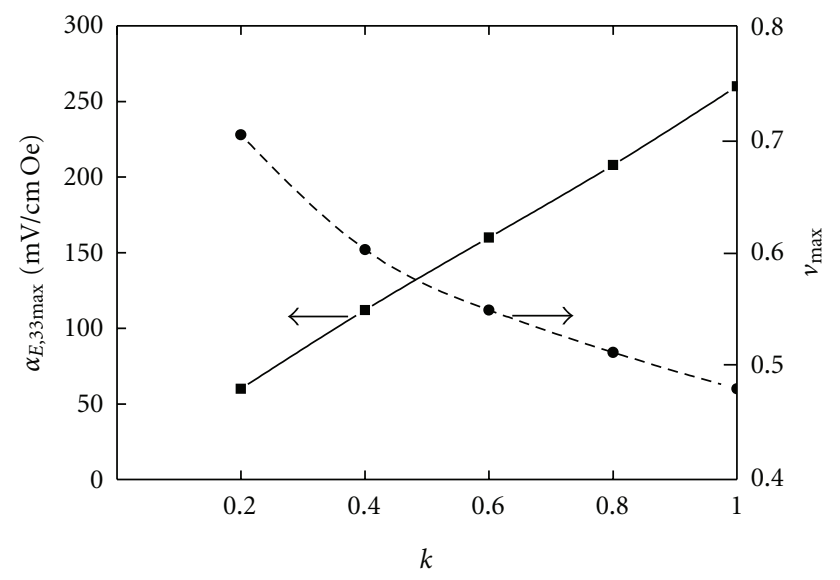

(b)

FIGURE 3: (a) Estimated dependence of longitudinal ME voltage coefficient on interface coupling $k$ and volume fraction $v$ for symmetric structure of CFO and PZT. (b) Variation with $k$ of maximum $\alpha_{E, 33}$ and the corresponding $v_{\max }$.

$v_{\max }$ shifts to PZT-rich compositions. Figure 3(b) shows the dependence of the maximum value in $\alpha_{E, 33}$ on $k$, where calculations are illustrated for various values of $v_{\max }$. With increasing $k$, a near-linear increase was found in the maximum value of $\alpha_{E, 33}$. For transverse fields, the maximum $\alpha_{E}$ is $40 \%$ higher than that of $\alpha_{E, 33}$. This is due to the strong parallel piezomagnetic coupling $q_{11}$ which determines $\alpha_{E}$, relative to that of $q_{31}$ which determines $\alpha_{E, 33}$.

Next, we consider the ME effect in CFO-PZT for the inplane longitudinal field orientation.

The most significant prediction of the present model is that the strongest ME coupling should occur for inplane longitudinal fields, as shown in Figure 4. One can easily see in Figure 4 that when the field is switched from longitudinal to in-plane longitudinal that the maximum value of the relevant $\mathrm{ME}$ coefficient increases by nearly an order of magnitude: $\alpha_{E, \max }=325 \mathrm{mV} /(\mathrm{cm} \mathrm{Oe})$ for the longitudinal orientation, whereas $\alpha_{E, 11}=3600 \mathrm{mV} /(\mathrm{cm} \mathrm{Oe})$ for the in-plane longitudinal. The $v$-dependence of $\alpha_{E, 11}$ reveals a rapid increase in the $\mathrm{ME}$ coefficient to a maximum value of $\alpha_{E, 11}=3600 \mathrm{mV} /(\mathrm{cm} \mathrm{Oe})$ for $v=0.11$, which is followed by a near-linear decrease with further increase of $v$. Such an enhancement in the in-plane longitudinal coefficient relative to the longitudinal one is understandable due to (i) the absence of demagnetizing fields in the in-plane configuration, and (ii) increased piezoelectric and piezomagnetic coupling coefficients compared to longitudinal fields. The down-shift in the value of $v_{\max }$ (from 0.5 to 0.6 for longitudinal and transverse fields to a much smaller value of 0.1 ) is due to the concentration dependence of the effective permittivity.

Another layered structure of importance is nickel ferrite (NFO)-PZT. Although NFO is a soft ferrite with a much smaller anisotropy and magnetostriction than CFO, efficient magnetomechanical coupling in NFO-PZT gives rise to ME voltage coefficients comparable to those of CFO-PZT. Using

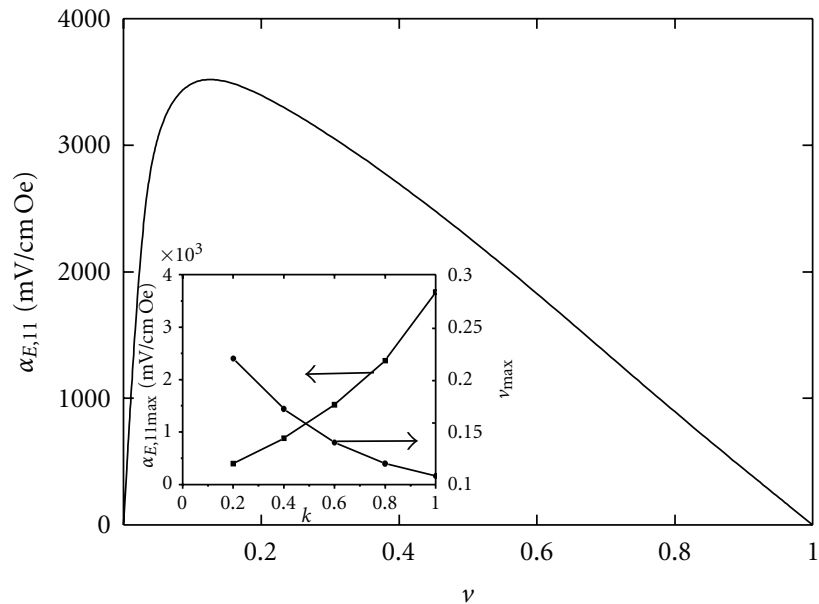

FIGURE 4: ME voltage coefficient for a perfectly bonded $(k=1)$ symmetric structure of CFO and PZT for in-plane longitudinal field orientation. The poling field and DC and AC magnetic and electric fields are in the sample plane and parallel to each other. Inset shows variation of maximum $\alpha_{E, 33}$ and the corresponding $v_{\max }$ with $k$.

the model presented in this chapter, we can estimate $\alpha_{E}$ for NFO-PZT for different field orientations and conditions, similar to that for CFO-PZT.

Finally, we consider composites that have lanthanum strontium manganites for the magnetostrictive phase. Lanthanum manganites with divalent substitutions have attracted considerable interest in recent years due to double exchange mediated ferromagnetism, metallic conductivity, and giant magnetoresistance [15]. The manganites are potential candidates for ME composites because of (i) high magnetostriction and (ii) metallic conductivity that eliminates the need for a foreign electrode at the interface. Figure 5 shows the longitudinal and transverse ME voltage 


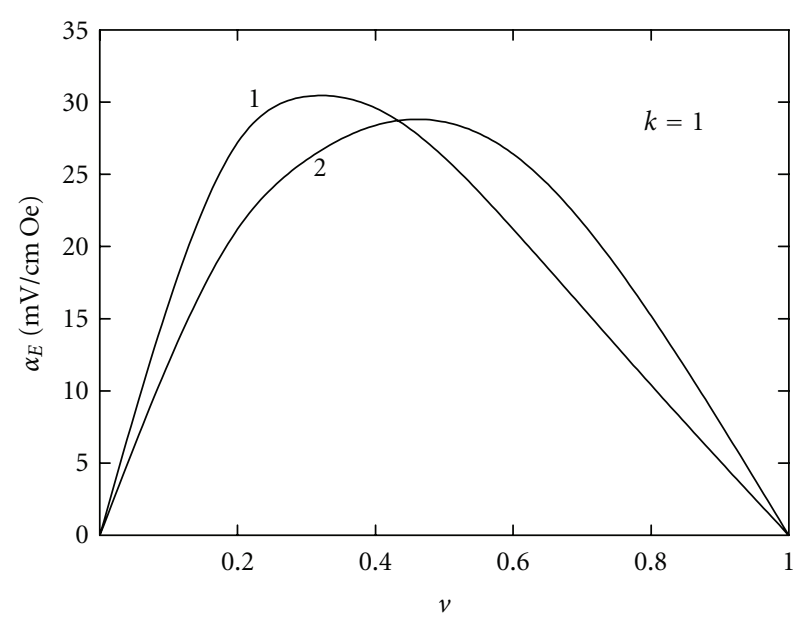

Figure 5: (1) Longitudinal and (2) transverse ME voltage coefficients as functions of PZT volume fraction for symmetric layered structure of $\mathrm{La}_{0.3} \mathrm{Sr}_{0.7} \mathrm{MnO}_{3}$ (LSMO) and PZT for interface coupling parameter $k=1$.

coefficients for unclamped $\mathrm{La}_{0.3} \mathrm{Sr}_{0.7} \mathrm{MnO}_{3}$ - (LSMO-) PZT laminate that assumes ideal coupling at the interface and no bending strain. In this case, the values of the ME coefficients are quite small compared to that of ferrite-PZT: this is due to weak piezomagnetic coefficients and compliances parameters for LSMO. The ME coefficient for in-plane longitudinal fields and the effects of clamping for different field orientations were similar in nature to those for ferrite-PZT layered structure, and thus are not discussed in any detail here.

It is important to compare the theoretical predictions, illustrated above, with experimental data. Let us consider first a bilayer of CFO-PZT taking into account the flexural deformations. Figure 6 shows $\alpha_{E}$ as a function of $v$. These data were obtained at low frequencies $(100-1000 \mathrm{~Hz})$. The desired volume fractions $v$ was achieved by careful control of the layer thickness. Data show an increase in $\alpha_{E}$ with $v$ until a maximum is reached. However, these data clearly demonstrated that the actual experimental value is an order of magnitude smaller than that predicted in Figures 2 and 3 (assuming $k=1$ ). It is, therefore, reasonable to compare the data with calculated values of $\alpha_{E}$ as a function of $v$ using a reduced interface coupling parameters of $k=0.1$ : in this case, agreement between theory and experiment can be seen, as shown in Figure 6. The key inference that can be made concerns the inherently poor interface coupling for CFOPZT, irrespective of sample synthesis techniques. We address possible causes for this poor coupling later in this section.

A third materials couples, LSMO-PZT, is considered in Figure 7, which shows $\alpha_{E}$ as a function of $v$ for longitudinal and transverse fields. The $\alpha_{E}$ values are the smallest amongst the three systems considered here. Calculated values assuming $k=1$ were found to be quite high compared to the data, rather it was found that nonideal values of $k=0.2$ gave reasonable agreement with the data. Thus, one can readily infer poor interfacial coupling in LSMO-PZT, similar to that for CFO-PZT.
Finally, we should comment on a possible cause of poor interfacial coupling for CFO-PZT and LSMO-PZT, and ideal coupling for NFO-PZT. The parameter $k$ can be expected to be sensitive to mechanical, structural, chemical, and electromagnetic parameters at the interface. We attribute unfavorable interface conditions in CFO-PZT and LSMOPZT to inefficient magnetomechanical coupling. The magnetomechanical coupling $k_{m}$ is given by $k_{m}=\left(4 \pi \lambda^{\prime} \mu_{r} / E\right)^{1 / 2}$; where $\lambda^{\prime}$ is the dynamic magnetostrictive constant and $\mu_{r}$ is the reversible permeability, and $E$ is Young's modulus. In ferrites, under the influence of a DC magnetic bias $H$ and AC magnetic field $\delta H$, domain wall motion and domain rotation contribute to the Joule magnetostriction and consequently to the effective linear piezomagnetic coupling. A key requirement for strong coupling is unimpeded domain wall motion and domain rotation. A soft ferrite with a high initial permeability (i.e., low anisotropy), such as NFO, will have key materials parameters favoring a high $k_{m}$, and consequently, strong ME effects. Measurements have shown that NFO has an initial permeability of 20 , whereas that of LSMO and CFO is 2-3. Thus, one can infer a plausible simple explanation of the near interfacial parameter for NFO-PZT is (in part) favorable domain motion.

In deriving the above expression, we assumed the electric field to be zero in magnetic phase since magnetostrictive materials that are used in the case under study have a small resistance compared to piezoelectric phase. Estimate of $\mathrm{ME}$ voltage coefficient for CFO-PZT layered structure gives $\alpha_{E, 33}$ $=325 \mathrm{mV} /(\mathrm{cm} \mathrm{Oe})$ providing that the bending strains are ignored. However, considering $\mathrm{CFO}$ as a dielectric results in $\alpha_{E, 33}=140 \mathrm{mV} /(\mathrm{cm} \mathrm{Oe})[16]$ while the experimental value does not exceed $74 \mathrm{mV} /(\mathrm{cm} \mathrm{Oe})$ [10]. We believe CFO should be considered as a conducting medium compared to dielectric PZT in the low-frequency region in accordance with our model. The discrepancy between theoretical estimates and data can be accounted for by features of piezomagnetic coupling in CFO and interface coupling of bilayer [13].

2.4. Bulk Composites. Design of new ME composites assumes the use of reliable theoretical models, allowing prediction of properties for various materials couples and over a range of laminate parameters. Manufacturing methods of all-ceramic composites are based on an initial mixing of starting powders batched in proportion to the composite volume fraction, followed by pressing and densification/sintering to a net-shape. Clearly, if the concentration of one of the constituent phases is small, then that phase will consist of isolated particles in a matrix. Following accepted classification nomenclature [17] this composite should be referred to as a 0-3 type, as one phase is isolated (i.e., connected in zero dimensions) and the second is interconnected in three dimensions. If the volume fraction of the secondary phase in the matrix is increased, and a percolation limit is reached, then it is classified as a 1-3-type composite. If the secondary phase then crosses that initial percolation limit, and subsequently begins to be interconnected in two dimensions, the composite connectivity is known as the 2-3 type. We mention these things at this time to make the point that the same ceramic 


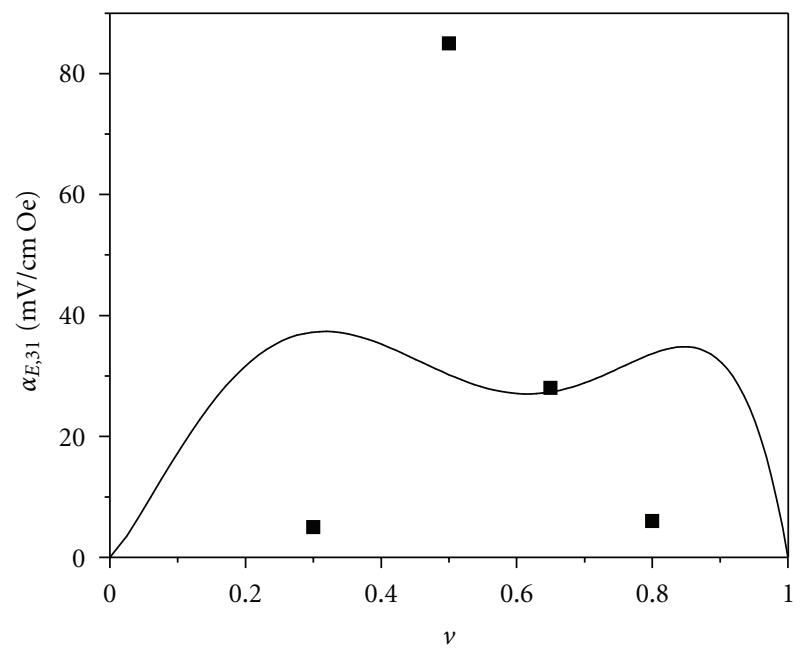

(a)

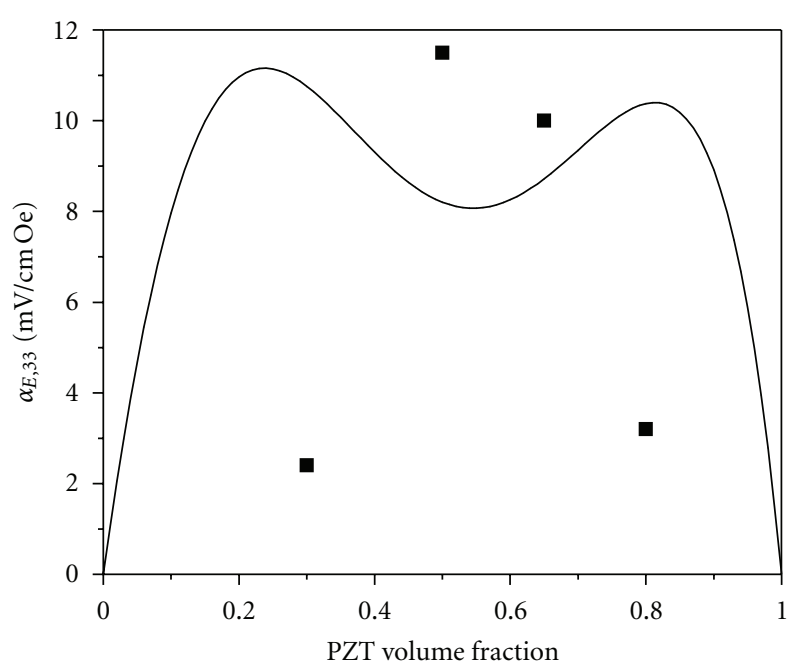

(b)

FIGURE 6: PZT volume fraction dependence of ME voltage coefficients of CFO-PZT bilayer. Solid lines are theory for $k=0.1$ and points are experiment.

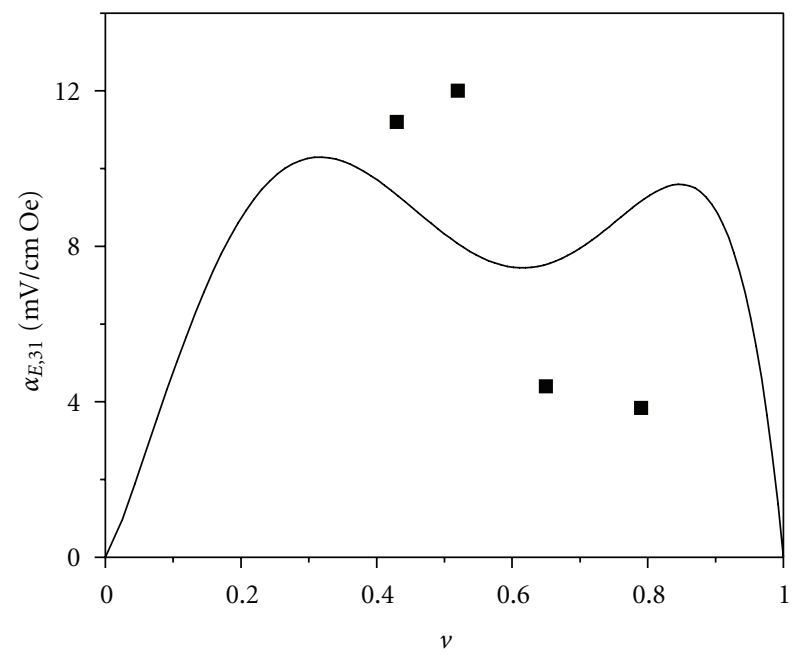

(a)

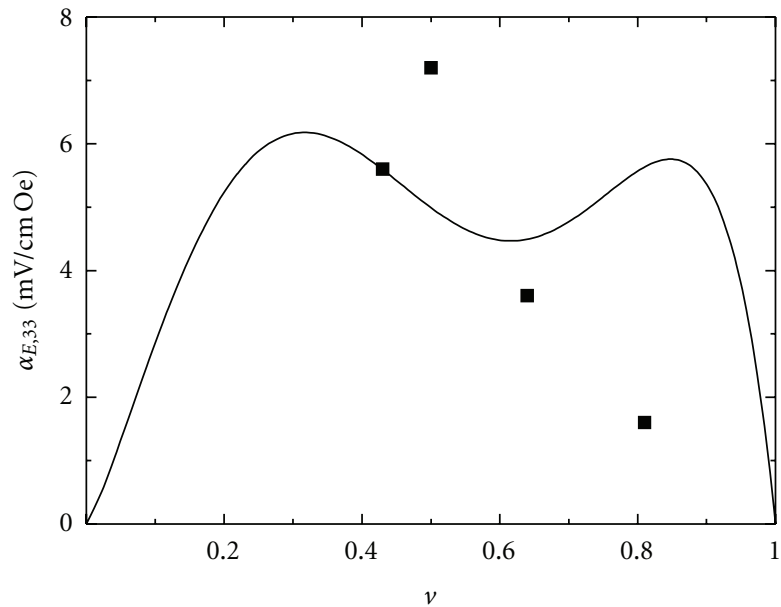

(b)

FIGURE 7: PZT volume fraction dependence of transverse (a) and longitudinal (b) ME voltage coefficients for LSMO-PZT bilayer: solid lines are the theory for $k=0.2$ and points are experiment.

manufacturing technology allows the fabrication of a wide range of relative volume fractions of the different phases in an all-ceramic composite, and consequently to various possible types of dimensional interconnectivities. Accordingly, it is very important to choose the correct method of calculation for effective constants of a composite at various relative volume fractions of components.

Unfortunately, exact solutions of three-dimensional problems related to the calculation of effective constants of inhomogeneous systems are unknown. Therefore, there is presently no precise structural classification of composites. Within the limited theory of heterogeneous systems of twophase composites, there are two principle approaches to approximate solutions: matrix systems and two-component mixtures, for which behavior of effective parameters depends on concentration continuously.
In the case of matrix systems, modification of the concentration from 0 to 1 does not change the qualitatively structure of the composite: at any concentration, one of the components must form a coherent matrix that contains isolated particles of the second component. The system always remains essentially noncentral, and matching formulas for an evaluation of effective constants give their continuous dependence on concentration in the entire range from 0 to 1 . We should note that the application of these formulas to the calculation of effective constants of composites is not always justified.

The case of two-component mixtures is characterized by a qualitative modification of the structure of the composite, as the concentration is changed. Such systems are characterized, as is well known, by critical concentrations at which point there are important property changes such as 


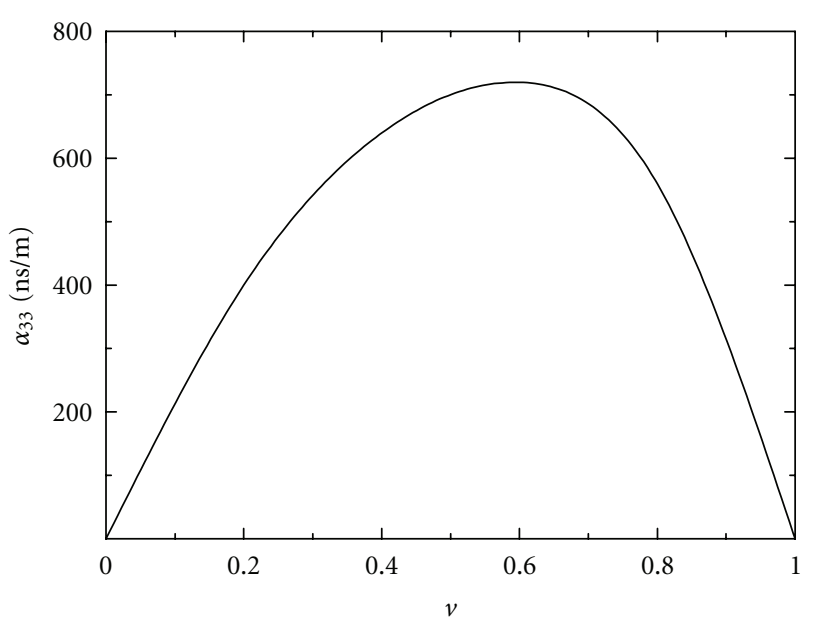

Figure 8: PZT volume fraction dependence of ME susceptibility for CFO-PZT composite with connectivity 3-0.

metal-insulator or rigid-plastic transformations. The metalinsulator transformation occurs in a composite consisting of an insulating and conductive phases. Assume that the insulating phase is initially the matrix and that the conductive one consists of isolated particles. In this case, initially the composite is insulating; however, when the percolation limit is crossed, the conducting particles form an interconnected conduction pathway, dramatically lowering the resistivity near a critical volume fraction. In the second example (rigid-plastic transformation), it is supposed that the composite is a mixture in which the elastic compliance of one of the constituent phases tends to infinity (e.g., a porous composite). This composite type possesses a critical concentration of the second phase, above which the rigid framework of the composite loses its stability. It should be straightforward to see that any bulk composites will have numerous effective materials properties, all of which change with relative phase volume fraction in a manner independent of other properties.

As an example, we consider a composite with a 30 type connectivity. Cubic models for ferrite-ferroelectric composites with a connectivity of 3-0 and $0-3$ have been considered by Harshe et al. [18]. Numerically, the ME coefficient is equal to the ratio of the electric field induced on the composite by an applied magnetic field: the ME coefficient is equal to $E_{3} / H_{3}$. It is necessary to realize that the magnetic field was applied only to the ferrite phase: that is, $E_{3} /{ }^{m} H_{3}$ where ${ }^{m} H_{3}$ is the local magnetic field on the ferrite phases which may exceed that applied to the entire composite. Harshe's study only considered the case of free cubic cells, and effective parameters of the composite in known model systems were not determined. However, in real composites, we must consider the case of nonfree cells. It is also very important to use any such model to predict the effective composite parameters. In the following section, we present a generalized model for ferrite-piezoelectric composites that allows one to define and predict the effective parameters of said composite using given conditions.

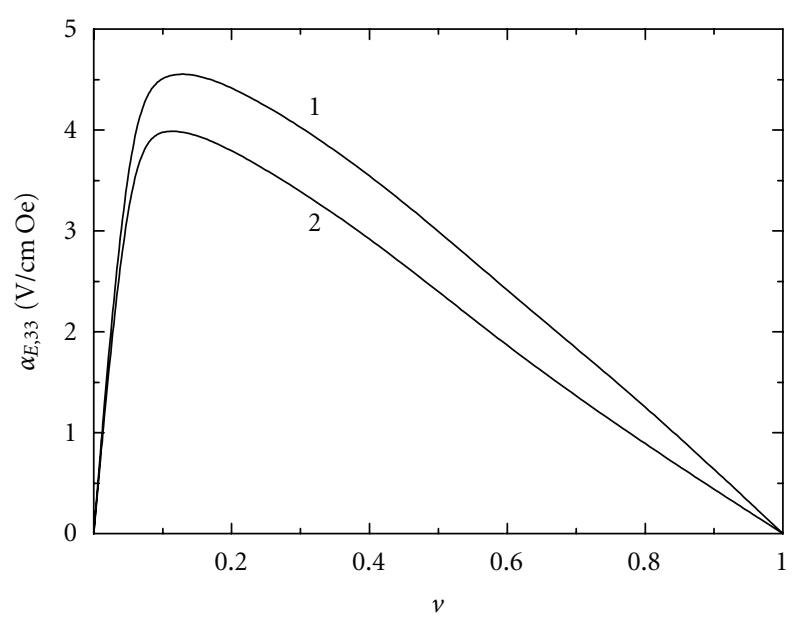

Figure 9: PZT volume fraction dependence of ME voltage coefficient for CFO-PZT composite with connectivity 3-0 according to model (1) [19] and model (2) [18] for $\alpha_{E, 33}=E_{3} /{ }^{m} H_{3}$ and material parameters from Table 1.

The properties of this ME composite will depend on the parameters of the corpuscles, and also on the terminal conditions. Now, let us suppose that the geometrical model for $\mathrm{ME}$ composites in this figure is miniaturized to fine scales. If the given cubic model ME composite is considered as a material consisting of consecutive and parallel connections of cubic cells with legs of unit length, then it is obvious by the definition of properties of a composite that it is possible to consider only one cubic cell rather than the entire ensemble of cells. The magnetostriction phase is enclosed by piezoelectric ones along different directions.

To derive the effective material parameters of a composite, we use an averaging method similar to that at consideration of layered structures. To account for the external forces that act on the cells in the cubic model, it is necessary to consider the clamped condition. Thus, to the components of the stress tensor, one must add additional external stress components. In addition, it is necessary to use boundary conditions of the type

$$
\begin{aligned}
& S_{1}=-s_{c 1} s_{11} T_{1}, \\
& S_{2}=-s_{c 2} s_{22} T_{2}, \\
& S_{3}=-s_{c 3} s_{33} T_{3},
\end{aligned}
$$

where $s_{c i}={ }^{c} s_{i i} / s_{i i}$ is the relative compliance of surroundings, and ${ }^{c} s_{i i}$ is effective compliance of composite.

PZT volume fraction dependence of effective ME susceptibility is shown in Figure 8 [19].

The dependence of the effective ME voltage coefficient, defined as $\alpha_{E, 33}=-\alpha_{33} / \varepsilon_{33}$, on the piezoelectric phase volume fraction can then be easily obtained, as shown in Figure 9. These graphical solutions then allow one to determine the piezoelectric and magnetostrictive phase volume fractions that yield maximum values for the effective ME susceptibility (Figure 8) and the ME voltage coefficient (Figure 9). 


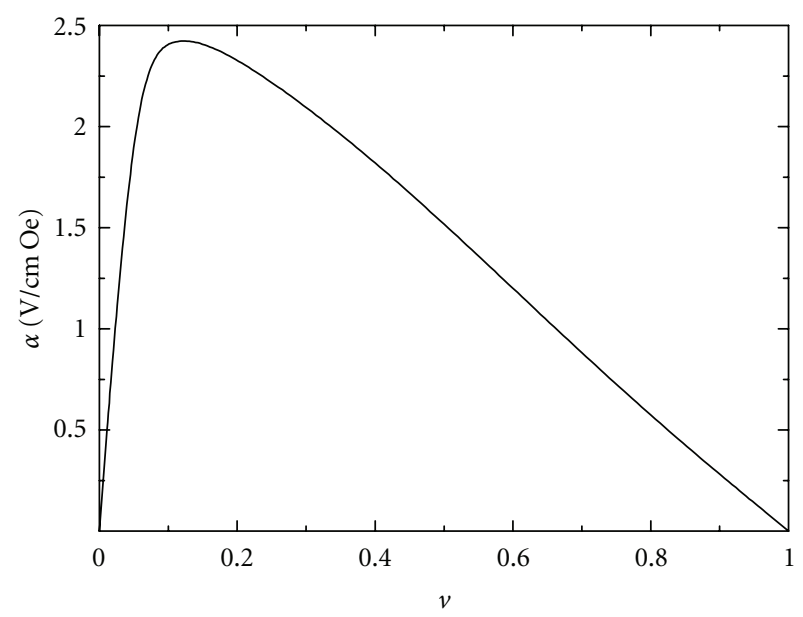

Figure 10: Concentration dependence of $\alpha_{E, 33}=E_{3} /{ }^{m} H_{3}$ for composite with material parameters from [19].

The values of the ME voltage coefficient in Figure 10 coincide with previously published data [18], demonstrating the usefulness of the predictions. As follows from Figure 10, the ME voltage coefficient was approximately $20 \%$ greater than that calculated from the experimental data using the model. This is explained by the fact that the internal (local) magnetic field in the ferrite component is considerably different than that of the externally applied magnetic field.

Measurements of the ME voltage coefficient have been performed for bulk composites of NFO-PZT, using the experimental methodology mentioned above. Data are shown in Figure 11 for the ME voltage coefficient as a function of the piezoelectric phase volume fraction. These measured values are much lower in magnitude than the theoretical ones predicted for the free composite condition. However, considering a clamped condition defined by the matching $s_{c 11}=s_{c 22}=s_{c 33}=0.3 s_{33}$, agreement between theory and experiment was found, as illustrated in Figure 11. These results indicate in real 0-3 ferrite-piezoelectric ceramic composites mixtures that the component phase grains are mechanically clamped by neighboring grains and by environmental boundary conditions.

\section{Modeling of Magnetoelectric Effect in Electromechanical Resonance Region}

3.1. Longitudinal Modes. In this section, we present a theory for the resonance enhancement of magnetoelectric (ME) interactions at frequencies corresponding to electromechanical resonance (EMR). Frequency dependence of ME voltage coefficients is obtained using the simultaneous solution of electrostatic, magnetostatic, and elastodynamic equations. The ME voltage coefficients are estimated from known material parameters (piezoelectric coupling, magnetostriction, elastic constants, etc.) of composite components. It is shown that the resonance enhancement of ME interactions is observed at frequencies corresponding to EMR and ME coupling in the EMR region exceeds the low-frequency value

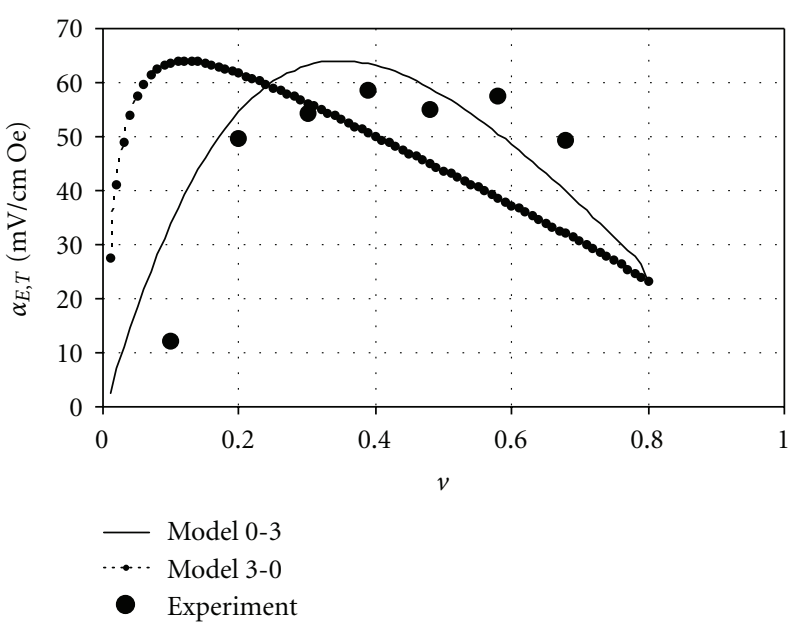

Figure 11: ME effect in bulk composite of NFO and PZT.

by more than an order of magnitude. It was found that the peak transverse ME coefficient at EMR is larger than the longitudinal one. The results of calculations obtained for a CFO-PZT composite are in good agreement with the experimental data.

The magnetoelectric (ME) effect in composites is caused by mechanically coupled magnetostrictive and piezoelectric subsystems: it is present in neither subsystem separately. Under magnetic field owing to the magnetostriction of the ferrite component, there are stresses which are elastically transmitted in the piezoelectric phase resulting in polarization changes via piezoelectricity. Because the ME effect in composites is due to mechanically coupled piezoelectric and magnetostrictive subsystems, it sharply increases in the vicinity of the electromechanical resonance (EMR) frequency [20-22].

Mechanical oscillations of an ME composite can be induced either by alternating magnetic or electric fields. If the length of the electromagnetic wave exceeds the spatial size of the composite by some orders of magnitude, then it is possible to neglect gradients of the electric and magnetic fields within the sample volume. Therefore, based on elastodynamics and electrostatics, the equations of medium motion are governed by $[5,6,23]$

$$
\bar{\rho} \frac{\partial^{2} u_{i}}{\partial t^{2}}=V \frac{\partial^{p} T_{i j}}{\partial x_{j}}+(1-V) \frac{\partial^{m} T_{i j}}{\partial x_{j}}
$$

where $u_{i}$ is the displacement vector component, $\bar{\rho}=$ $V^{p} \rho+(1-v)^{m} \rho$ is the average mass density, $V$ is the ferroelectric volume fraction, $p \rho$ and ${ }^{m} \rho, p^{p} T_{i j}$, and ${ }^{m} T_{i j}$ are the densities and stress tensor components of ferroelectric and ferrite, correspondingly.

As an example, we consider a composite that has the form of a narrow plate which has a length $L$. Solution of (12) by use of elasticity equations, appropriate boundary conditions 


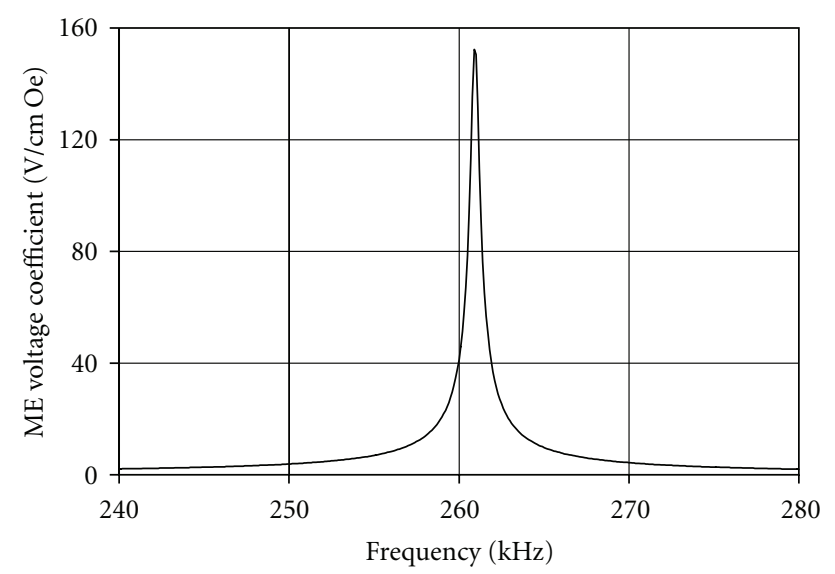

Figure 12: Frequency dependence of $\alpha_{E, 13}$ for the bilayer of CFO and PZT with length of $7.3 \mathrm{~mm}$ and PZT volume fraction 0.5 .

and open circuit condition allow one to find the ME voltage coefficient for transverse fields orientation [23]:

$$
\alpha_{E, 31}=\frac{2^{p} d_{31}{ }^{m} g_{11} \mu_{\mathrm{eff}}{ }^{p} s_{11} v(1-v) \tan (k L / 2)}{s_{2}\left({ }^{p} d_{31}^{2}-{ }^{p} s_{11}{ }^{p} \varepsilon_{33}\right) k L-2^{p} d_{31}^{2} v^{m} s_{11}^{B} \tan (k L / 2)},
$$

where $k=\omega \sqrt{\bar{\rho}\left[V /{ }^{p} s_{11}+(1-V) /{ }^{m} s_{11}\right]^{-1}}, \mu_{\text {eff }}$ is effective permeability of piezomagnetic layer, and $s_{1}=V^{m} s_{11}+(1-$ V) ${ }^{p} s_{11}$.

The roots of the denominator in (13) define the maxima in the frequency dependence of the ME voltage coefficient. As one can see from (13), the value of the ME coefficient under applied fields is directly proportional to the product of piezoelectric $d_{31}$ and piezomagnetic $g_{11}$ modules. To take into consideration the energy loss, we set $\omega$ equal to $\omega^{\prime}-i \omega^{\prime \prime}$ with $\omega^{\prime \prime} / \omega^{\prime}=10^{-3}$. The resonance enhancement of $\mathrm{ME}$ voltage coefficient for the bilayer is obtained at antiresonance frequency. $\mathrm{ME}$ voltage coefficient, $\alpha_{E, 13}$ increases with increasing PZT volume fraction, attains a peak value for $v=$ 0.5, and then drops with increasing $v$ as in Figure 12.

3.2. Bending Modes. A key drawback for ME effect at longitudinal modes is that the frequencies are quite high, on the order of hundreds of $\mathrm{kHz}$, for nominal sample dimensions. The eddy current losses for the magnetostrictive phase can be quite high at such frequencies, in particular for transition metals and alloys and earth rare alloys such as Terfenol-D, resulting in an inefficient magnetoelectric energy conversion. In order to reduce the operating frequency, one must therefore increase the laminate size that is inconvenient for any applications. An alternative for getting a strong ME coupling is the resonance enhancement at bending modes of the composite. The frequency of applied AC field is expected to be much lower compared to longitudinal acoustic modes. Recent investigations have showed a giant $\mathrm{ME}$ effect at bending modes in several layered structures [14, 24, 25]. In this section, we focus our attention on theoretical modeling of ME effects at bending modes [14].
An in-plane bias field is assumed to be applied to magnetostrictive component to avoid the demagnetizing field. The thickness of the plate is assumed to be small compared to remaining dimensions. Moreover, the plate width is assumed small compared to its length. In that case, we can consider only one component of strain and stress tensors in the EMR region. The equation of bending motion of bilayer has the form

$$
\nabla^{2} \nabla^{2} w+\frac{\rho b}{D} \frac{\partial^{2} w}{\partial \tau^{2}}=0
$$

where $\nabla^{2} \nabla^{2}$ is biharmonic operator, $w$ is the deflection (displacement in $z$-direction), $t$ and $\rho$ are thickness and average density of sample, $b={ }^{p} t+{ }^{m} t, \rho=\left({ }^{p} \rho p_{t}+{ }^{m} \rho{ }^{m} t\right) / b, p \rho$, ${ }^{m} \rho$, and ${ }^{p} t,{ }^{m} t$, are densities and thicknesses of piezoelectric and piezomagnetic, correspondingly, and $D$ is cylindrical stiffness.

The boundary conditions for $x=0$ and $x=L$ have to be used for finding the solution of above equation. Here $L$ is length of bilayer. As an example, we consider the plate with free ends. At free end, the turning moment $M_{1}$ and transverse force $V_{1}$ equal zero: $M_{1}=0$ and $V_{1}=0$ at $x=0$ and $x=L$, where $M_{1}=\int_{A} z T_{1} d z_{1}, V_{1}=\left(\partial M_{1}\right) / \partial x$, and $A$ is the cross-sectional area of the sample normal to the $x$-axis. We are interested in the dynamic ME effect; for an AC magnetic field $H$ applied to a biased sample, one measures the average induced electric field and calculates the $\mathrm{ME}$ voltage coefficient. Using the open circuit condition, the ME voltage coefficient can be found as

$$
\alpha_{E, 31}=\frac{E_{3}}{H_{1}}=-\frac{\int_{z_{0}-}^{z_{0}}{ }^{p}{ }^{p} E_{3} d z}{t H_{1}{ }^{p} \varepsilon_{33}},
$$

where $E_{3}$ and $H_{1}$ are the average electric field induced across the sample and applied magnetic field. The energy losses are taken into account by substituting $\omega$ for complex frequency $\omega^{\prime}+i \omega^{\prime \prime}$ with $\omega^{\prime \prime} / \omega^{\prime}=10^{-3}$.

As an example, we apply (14) to the bilayer of permendur and PZT. Figure 13 shows the frequency dependence of ME voltage coefficient at bending mode for free-standing bilayer with length $9.15 \mathrm{~mm}$ and thickness $3.22 \mathrm{~mm}$ for PZT volume fraction 0.67 . Graph of $\alpha_{E, 31}$ reveals a giant value $\alpha_{E, 31}=6.6 \mathrm{~V} / \mathrm{cm}$ Oe and resonance peak lies in the infralow frequency range. Figure 14 reveals the theoretical and measured frequency dependencies of transverse $\mathrm{ME}$ voltage coefficients for a permendur-PZT bilayer that is free to bend at both ends.

According to our model, there is a strong dependence of resonance frequency on boundary conditions. The lowest resonance frequency is expected for the bilayer clamped at one end. One expects bending motion to occur at decreasing frequencies with increasing bilayer length or decreasing thickness.

\section{Conclusions}

In this manuscript, a generalized theoretical model for lowfrequency ME effects in layered composites was discussed. 


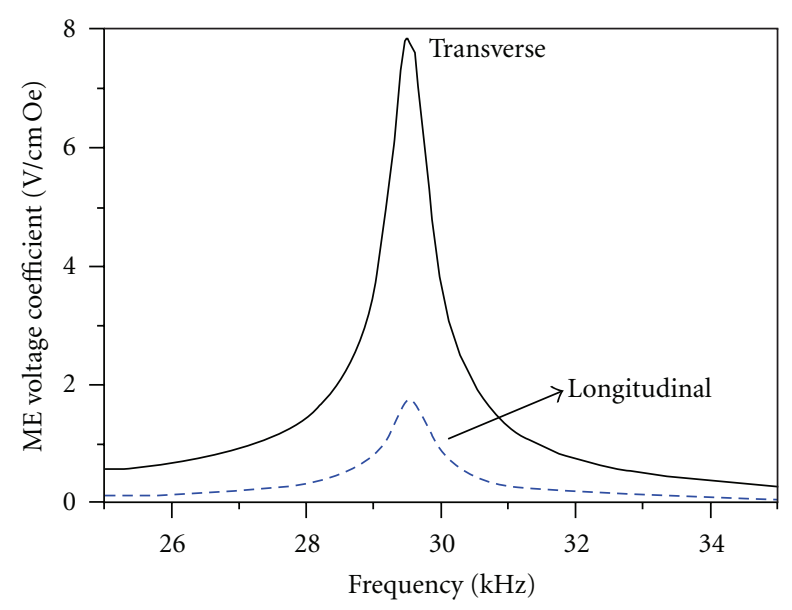

FIGURE 13: Frequency dependence of longitudinal and transverse ME voltage coefficients for a bilayer of permendur and PZT showing the resonance enhancement of ME interactions at the bending mode frequency. The bilayer is free to bend at both ends. The sample dimensions are $L=9.2 \mathrm{~mm}$ and total thickness $t=0.7 \mathrm{~mm}$ and the PZT volume fraction $v=0.6$.

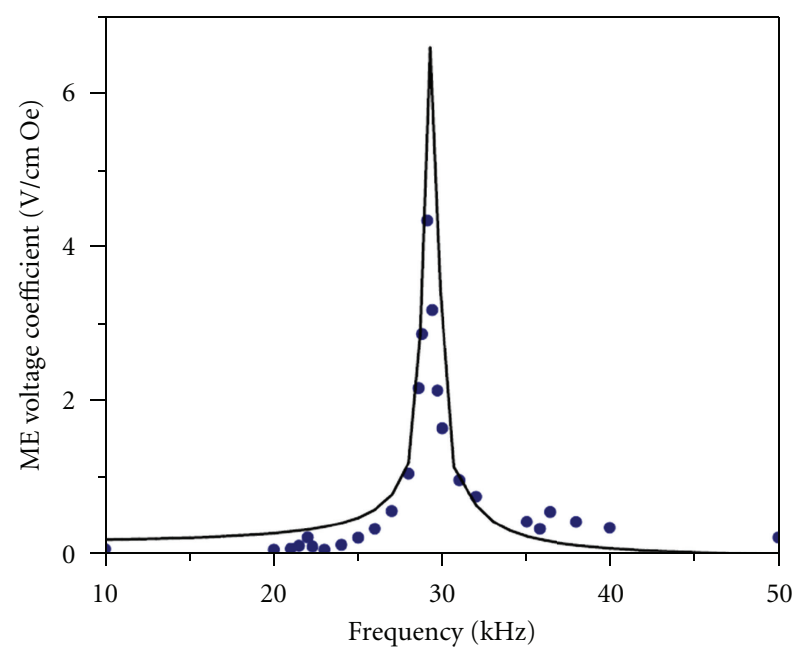

Figure 14: Theoretical (line) and measured (circles) frequency dependence of transverse ME voltage coefficients for a permendurPZT bilayer that is free to bend at both ends and with $v=0.67$.

To describe the composite's physical properties, the exact solution of elastostatic and electrostatic equations were obtained. Expressions for the ME susceptibility and ME voltage coefficient were derived as functions of an interface coupling parameter, constituent phase material parameters, and relative volume fractions of phases. Longitudinal, transverse, and in-plane cases were all considered properties. For a bilayer that is an asymmetric structure, the influence of flexural deformations of sample on ME output was estimated.

Predictions of the ME effect for various model composite systems were given including CFO-PZT and lanthanum strontium manganite-PZT. It was shown that ME effect in ferrite-PZT systems is maximum for in-plane magnetic and electric fields. The theoretical estimates of ME parameters were compared with experimental data.

The generalized theory allows for modeling of the lowfrequency ME effect in bulk composites. To describe these low-frequency composite properties, an effective medium method was used. Calculation of the ME susceptibility, and $\mathrm{ME}$ voltage coefficient were performed as functions of volume fractions and component parameters. Composites with connectivity types 3-0 and 0-3 were considered. Larger ME coefficients were found for 3-0 composites with magnetic and/or electric fields applied along the longitudinal direction. For composites of CFO-PZT, values as high as $4 \mathrm{~V} /(\mathrm{cm} \mathrm{Oe})$ were predicted for the longitudinal ME voltage coefficient. For the transverse fields orientation, ME effect was found to be in 2-3.5-times smaller than that for longitudinal orientation. Furthermore, clamping was shown to significantly reduce the ME effect.

We presented a theory for the resonance enhancement of $\mathrm{ME}$ interactions at frequencies corresponding to EMR. Frequency dependence for ME voltage coefficients are obtained using the simultaneous solution of electrostatic, magnetostatic, and elastodynamic equations. The ME effect at bending mode in a bilayer is shown to be dependent on boundary conditions. A giant ME interaction at the lowest frequency is predicted for a bilayer fixed at one end and is free to vibrate at the other end. The ME voltage coefficients are estimated from known material parameters (piezoelectric coupling, magnetostriction, elastic constants, etc.) of composite components. It is shown that the ME coupling in the EMR region exceeds the low-frequency value by more than an order of magnitude.

\section{References}

[1] D. N. Astrov, "Magnetoelectric effect in chromium oxide," Journal of Experimental and Theoretical Physics, vol. 13, p. 729, 1961.

[2] J. van den Boomgaard, A. M. J. G. van Run, and J. van Suchtelen, "Piezoelectric-piezomagnetic composites with magnetoelectric effect," Ferroelectrics, vol. 14, p. 727, 1976.

[3] J. Ryu, A. V. Carazo, K. Uchino, and H. E. Kim, "Piezoelectric and magnetoelectric properties of lead zirconate titanate/Niferrite particulate composites," Journal of Electroceramics, vol. 7, no. 1, pp. 17-24, 2001.

[4] V. M. Laletin and G. Srinivasan, "Magnetoelectric effects in composites of nickel ferrite and barium lead zirconate titanate," Ferroelectrics, vol. 280, pp. 177-185, 2002.

[5] C.-W. Nan, M. I. Bichurin, S. Dong, D. Viehland, and G. Srinivasan, "Multiferroic magnetoelectric composites: historical perspective, status, and future directions," Journal of Applied Physics, vol. 103, Article ID 031101, 35 pages, 2008.

[6] E. Asher, "The interaction between magnetization and polarization: phenomenological symmetry consideration," Journal of the Physical Society of Japan, vol. 28, no. 7, 1969.

[7] G. Srinivasan, E. T. Rasmussen, J. Gallegos, R. Srinivasan, Yu. I. Bokhan, and V. M. Laletin, "Magnetoelectric bilayer and multilayer structures of magnetostrictive and piezoelectric oxides," Physical Review B - Condensed Matter and Materials Physics, vol. 64, no. 21, pp. 2144081-2144086, 2001.

[8] S. Dong, J. Zhai, F. Bai, J. -F. Li, and D. Viehland, "Pushpull mode magnetostrictive/piezoelectric laminate composite 
with an enhanced magnetoelectric voltage coefficient," Applied Physics Letters, vol. 87, no. 6, article 062502, 2005.

[9] S. Dong, J. Zhai, J. Li, and D. Viehland, "Near-ideal magnetoelectricity in high-permeability magnetostrictive/ piezofiber laminates with a (2-1) connectivity," Applied Physics Letters, vol. 89, no. 25, article 252904, 2006.

[10] G. Harshe, J. P. Dougherty, and R. E. Newnham, "Theoretical modelling of multilayer magnetoelectric composites," International Journal of Applied Electromagnetics in Materials, vol. 4, no. 2, pp. 145-159, 1993.

[11] M. I. Bichurin, V. M. Petrov, and G. Srinivasan, "Modeling of magnetoelectric effect in ferromagnetic/piezoelectric multilayer composites," Ferroelectrics, vol. 280, pp. 165-175, 2002.

[12] M. I. Bichurin, V. M. Petrov, and G. Srinivasan, "Theory of low-frequency magnetoelectric effects in ferromagneticferroelectric layered composites," Journal of Applied Physics, vol. 92, no. 12, pp. 7681-7683, 2002.

[13] M. I. Bichurin, V. M. Petrov, and G. Srinivasan, "Theory of low-frequency magnetoelectric coupling in magnetostrictivepiezoelectric bilayers," Physical Review B, vol. 68, no. 5, Article ID 054402, pp. 544021-5440213, 2003.

[14] V. M. Petrov, G. Srinivasan, M. I. Bichurin, and T. A. Galkina, "Theory of magnetoelectric effect for bending modes in magnetostrictive- piezoelectric bilayers," Journal of Applied Physics, vol. 105, no. 6, Article ID 063911, 2009.

[15] A. P. Ramirez, "Colossal magnetoresistance," Journal of Physics Condensed Matter, vol. 9, no. 39, pp. 8171-8199, 1997.

[16] I. A. Osaretin and R. G. Rojas, "Theoretical model for the magnetoelectric effect in magnetostrictive/ piezoelectric composites," Physical Review B, vol. 82, no. 17, Article ID 174415, 2010.

[17] R. E. Newnham, D. P. Skinner, and L. E. Cross, "Connectivity and piezoelectric-pyroelectric composites," Materials Research Bulletin, vol. 13, no. 5, pp. 525-536, 1978.

[18] G. Harshe, J. P. Dougherty, and R. E. Newnham, "Theoretical modelling of 3-0/0-3 magnetoelectric composites," International Journal of Applied Electromagnetics in Materials, vol. 4, no. 2, pp. 161-171, 1993.

[19] V. M. Petrov, M. I. Bichurin, V. M. Laletin, N. Paddubnaya, and G. Srinivasan, Magnetoelectric Interaction Phenomena in Crystals-NATO Science Series II, vol. 164, Kluwer Academic Publishers, London, UK, 2004, Edited By, M. Fiebig, V. V. Eremenko, and I. E. Chupis.

[20] G. Srinivasan, C. P. de Vreugd, V. M. Laletin et al., "Resonant magnetoelectric coupling in trilayers of ferromagnetic alloys and piezoelectric lead zirconate titanate: the influence of bias magnetic field," Physical Review B, vol. 71, no. 18, article 184423, 2005.

[21] M. I. Bichurin, D. A. Filippov, V. M. Petrov, V. M. Laletsin, N. Paddubnaya, and G. Srinivasan, "Resonance magnetoelectric effects in layered magnetostrictive-piezoelectric composites," Physical Review B - Condensed Matter and Materials Physics, vol. 68, no. 13, Article ID 132408, pp. 1324081-1324084, 2003.

[22] V. M. Petrov, M. I. Bichurin, and G. Srinivasan, "Electromechanical resonance in ferrite-piezoelectric nanopillars, nanowires, nanobilayers, and magnetoelectric interactions," Journal of Applied Physics, vol. 107, no. 7, Article ID 073908, 2010.

[23] M. I. Bichurin, V. M. Petrov, S. V. Averkin, and A. V. Filippov, "Electromechanical resonance in magnetoelectric layered structures," Physics of the Solid State, vol. 52, no. 10, pp. 2116-2122, 2010.

[24] V. M. Petrov, M. I. Bichurin, V. V. Zibtsev, S. K. Mandal, and G. Srinivasan, "Flexural deformation and bending mode of magnetoelectric nanobilayer," Journal of Applied Physics, vol. 106, no. 11, Article ID 113901, 2009.

[25] M. I. Bichurin, V. M. Petrov, S. V. Averkin, and E. Liverts, "Present status of theoretical modeling the magnetoelectric effect in magnetostrictive-piezoelectric nanostructures. Part I: low frequency and electromechanical resonance ranges," Journal of Applied Physics, vol. 107, no. 5, Article ID 053904, 2010. 

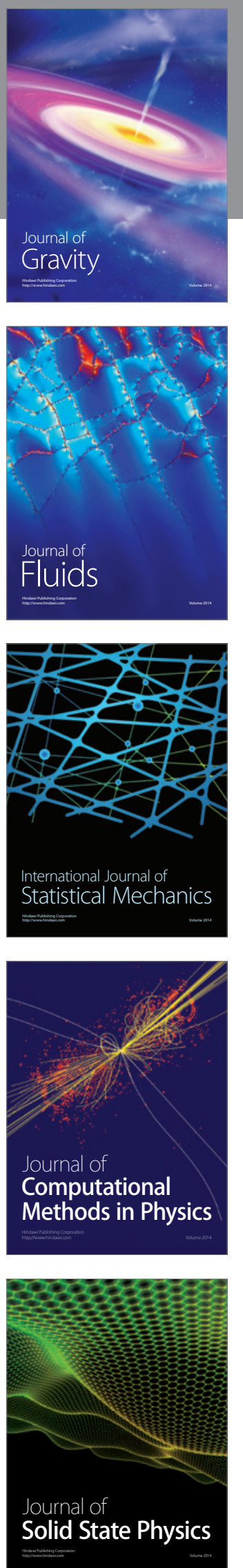

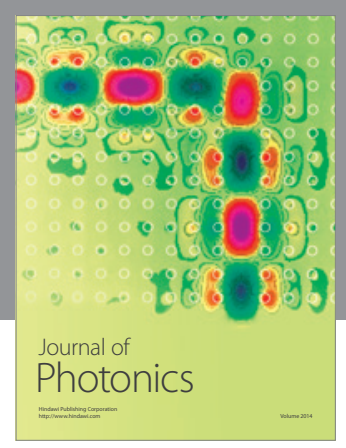

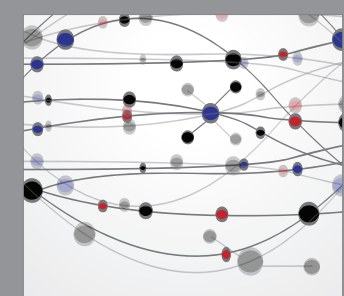

The Scientific World Journal
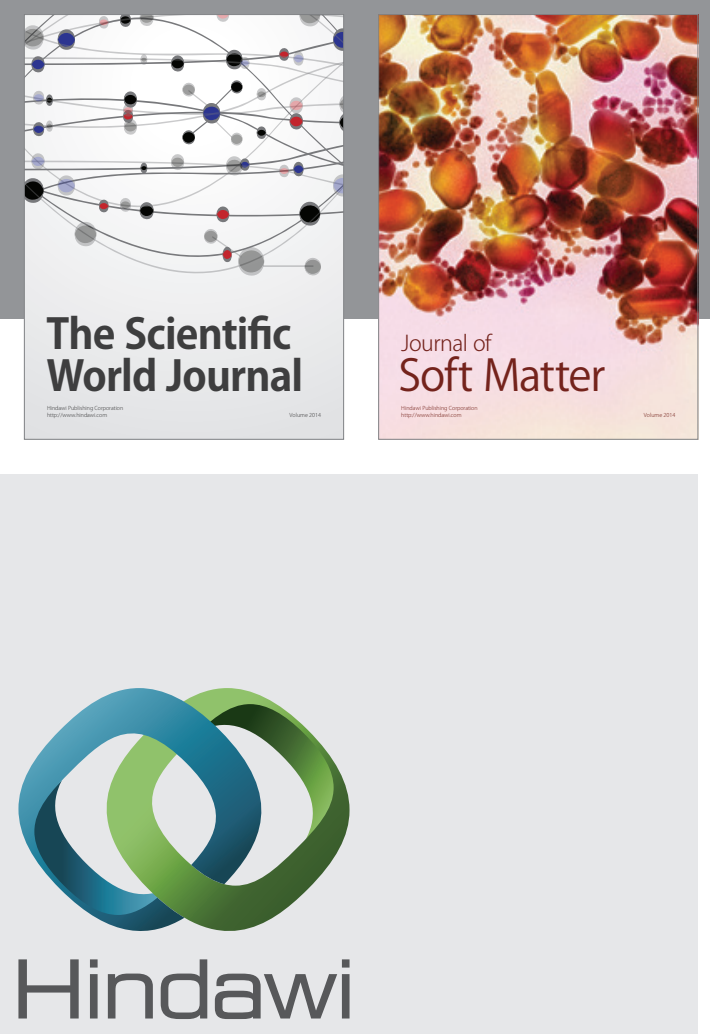

Submit your manuscripts at

http://www.hindawi.com
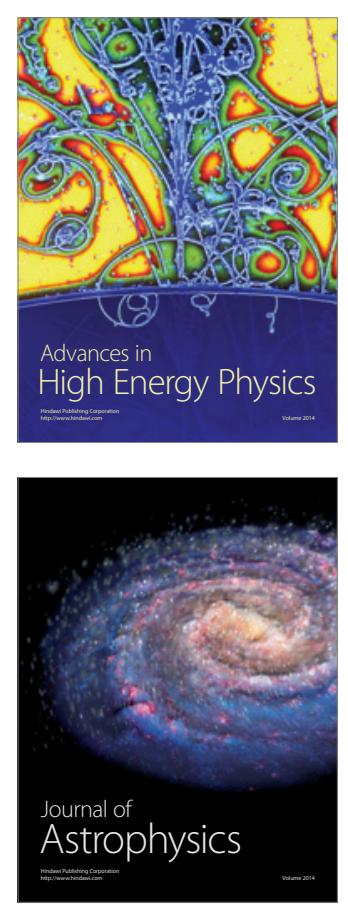
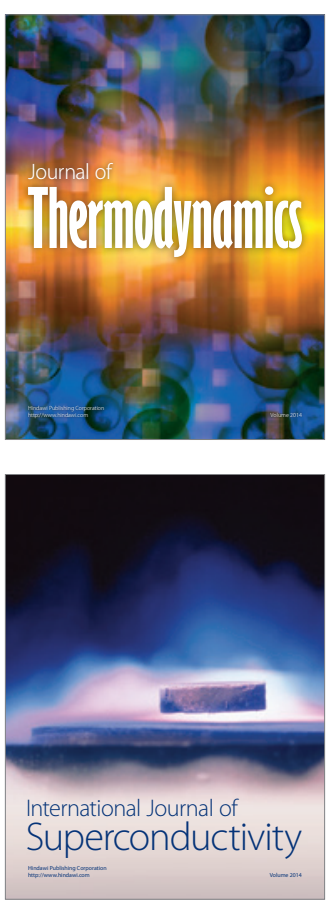
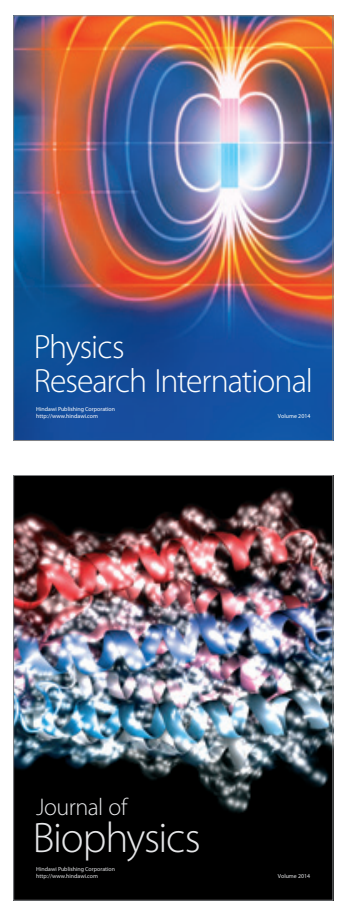
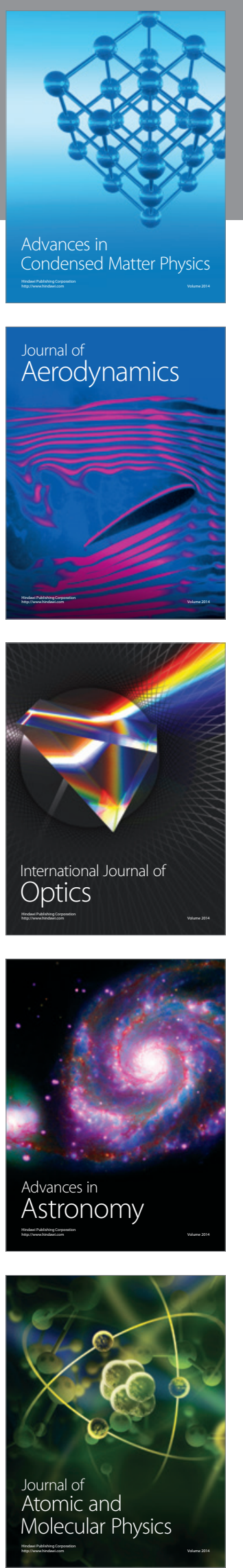